\title{
Research on License Plate Recognition of Chinese Vehicle Based on GWO-SVM Algorithm
}

\author{
Hao Ding, Jianguo Shen ${ }^{+}$ \\ College of Physics and Electronic Information Engineering \\ Zhejiang Normal University, Jinhua, China
}

\begin{abstract}
License Plate Recognition (LPR) technology has been widely used in traffic management system. In order to improve the efficiency of traditional LPR, this paper proposes a lightweight LPR algorithm based on Support Vector Machine (SVM) model with Grey Wolf Optimization (GWO) algorithm. GWO algorithm is used to seek the optimal parameters of the penalty factor and kernel parameter of SVM, which improves the accuracy of license plate character recognition. Besides, Gaussian filtering and grey level stretching are introduced for image preprocessing to enhance the quality of the gray level license plate image. Experiment results show that the recognition accuracy of the proposed character recognition model can reach more than 95\%. Compared with state-of-the-art LPR models using SVM, this algorithm is much faster on iteration.
\end{abstract}

Keywords: license plate recognition; Support Vector Machine; Grey Wolf Optimization algorithm; character recognition.

\section{Introduction}

Despite mature License Plate Recognition (LPR) systems available in the market, the problem of LPR is considered as a hot research issue [1]. Many effective LPR methods have been developed. For example, Da Li et al. [2] presented a new Convolutional Neural Network $(\mathrm{CNN})$ based character recognition algorithm and extracted the Chinese license plate characteristics more effectively. However, the algorithm itself relies on a large data set, which has high requirements on hardware and the complex network structure is not suitable for fast identification. In [3], the author discussed a MLP-SVM approach which has recognized all the characters of a license plate with high-accuracy in an average time of $57.4 \mathrm{~ms}$. But the algorithm combines a number of different SVMs, the structure is complex. And in [4], Yu et al. also developed a SVMbased algorithm combining Chinese character structure with Histogram of Oriented Gradient (HOG) features so as to improve the accuracy of LPR. Whereas, due to the influence of SVM default parameters, there is room for optimization improvement. In order to solve different problems such as light variation and occlusion, Khan et al. proposed a LPR system using entropy-based feature selection approach with SVM. The approach is an effort to deal under such conditions by incorporating multiple features extraction and fusion [5]. Nonetheless, it also ignored the influence of SVM parameters on recognition performance. Also, there are many kinds of LPR algorithms, including traditional recognition methods based on template matching algorithms, recognition methods based on feature point matching, hidden Markov random model recognition methods, Support Vector Machine (SVM) recognition model methods and artificial neural network methods. Considering that SVM learns through the optimal hyperplane, it has the characteristics of simple system structure, global optimality, no need of too much prior knowledge, and fast recognition speed. This paper uses SVM as the basic recognition framework. To solve the problem of parameter selection, Grey Wolf Optimization (GWO) [6] algorithm is introduced into LPR system based on SVM in this paper. In

\footnotetext{
+ Corresponding author. Tel:: 13738936751

E-mail address: shenjianguo981@163.com.
} 
addition, the proposed algorithm is light weight, which is suitable for the field of low-cost hardware applications.

\section{The proposed Method}

The general flow of license plate recognition is shown in fig.1. It includes five steps: (1) image preprocessing, (2) license plate location, (3) plate rectangular correction, (4) plate character segmentation, and (5) character recognition.

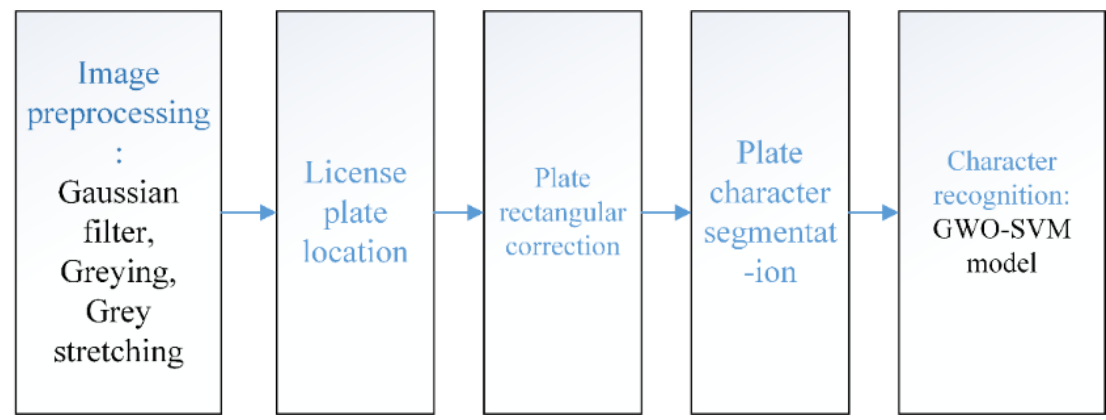

Fig. 1: The general flow of license plate recognition

The proposed method mainly focuses on the image pre-processing and character recognition. During the pre-processing step, the gray level stretch operation is introduced to enhance the gray level image after Gaussian filtering. And in the character recognition step, GWO algorithm is used to seek the optimal parameters of the penalty factor and kernel parameter of the classifier which is based on SVM. Then the character recognizer is trained with the best parameters.

\subsection{Image Preprocessing}

In the process of traditional license plate recognition, Gaussian filter is usually used to reduce the noise of color image. The Gaussian distribution and the weighted average greying value are expressed as:

$$
\begin{gathered}
G(x, y)=\frac{1}{2 \pi \sigma^{2}} e^{-\frac{x^{2}+y^{2}}{2 \sigma^{2}}} \\
F(i, j)=0.299 R(i, j)+0.579 G(i, j)+0.114 B(i, j)
\end{gathered}
$$

Where $G(x, y)$ is defined as the distribution value of the Gaussian function in two-dimensional space, and $\sigma$ is the standard deviation of its distribution. In two-dimensional space, the contours of the curved surface generated by this formula are concentric circles distributed normally from the center. In formula (2), where $F(i, j)$ is defined as the pixel grey value, $R(i, j), G(i, j)$ and $B(i, j)$ are the three channels for reading RGB component value.

However, there are problems of blurring the image and reducing the contrast of the image after the Gaussian filtering. In order to solve this problem, We introduce the gray stretching method to improve the quality of the gray image after the greying of the license plate image. Grayscale stretching expands the histogram of the image to fill the whole gray level range, which is helpful to enhance the contrast of the gray level image of the license plate.

Assume that the grayscale image is $F(i, j)$, the grayscale stretch image is shown in equation(3):

$$
G(i, j)=\left[\frac{255}{m-n}\right] \cdot[F(i, j)-n]
$$

Where $m$ is defined as the maximum gray level of the input gray image, and $n$ is defined as the minimum gray level of the input gray image.

\subsection{License Plate Character Recognition}

The SVM algorithm has been widely used in LPR field as the classifier of license plate character recognition. According to the SVM model, the nonlinear classification problem can be converted into the following formula: 


$$
\begin{gathered}
R(\omega)=\min \frac{1}{2}\|\omega\|^{2}+c \sum_{i=1}^{n}\left(\xi_{i}\right), \\
\text { s.t. } y_{i}\left(\omega^{T} x_{i}+b\right) \geq 1-\xi_{i}, \xi_{i} \geq 0, i=1,2, \ldots, n
\end{gathered}
$$

Where $\omega$ is defined as weight vector, $c$ is defined as penalty factor, and $\xi_{i}$ is defined as nonnegative relaxation variable. In addition, Radial Basis Function (RBF) is generally used as the kernel function to map the nonlinear problem to high-dimensional space, and its expression is:

$$
K\left(x_{i}, y_{j}\right)=e^{\left(-\frac{\left\|x_{i}-x_{j}\right\|^{2}}{2 g^{2}}\right)}
$$

According to the classical support vector machine algorithm, penalty factor $\mathrm{c}$ and kernel function RBF parameter $g$ have a great impact on character recognition. Therefore, Grey Wolf Optimization Algorithm is used to find the best combination of parameters suitable for Chinese license plate character recognition. GWO Algorithm is a meta heuristic algorithm based on population, which mainly realizes the purpose of parameter optimization by simulating the process of tracking, surrounding and attacking prey of wolves. In the GWO, the wolf group model is classified as the optimal solution represents $\alpha$, the sub optimal solution represents $\beta$, the third optimal solution represents $\delta$ and the rest solutions represent $\omega$. Its mathematical model for rounding up prey is:

$$
\begin{gathered}
D=\left|C \cdot X_{p}(t)-X(t)\right| \\
X(t+1)=X_{p}(t)-A \cdot D \\
C=2 \cdot r_{1} \\
A=2 a \cdot r_{2}-a
\end{gathered}
$$

Where $t$ is the number of iterations of the optimization algorithm, $X_{p}$ is the target position vector, and $X$ is the grey Wolf position vector, and $D$ is the distance between the grey Wolf and the target, $A$ and $C$ are the parameter vectors. After surrounding the prey, the wolves updated their positions using the following mathematical model:

$$
\begin{gathered}
D_{\alpha}=\left|C_{1} \cdot X_{\alpha}(t)-X(t)\right| \\
D_{\beta}=\left|C_{2} \cdot X_{\beta}(t)-X(t)\right| \\
D_{\delta}=\left|C_{3} \cdot X_{\delta}(t)-X(t)\right| \\
X_{1}=X_{\alpha}-A_{1} \cdot D_{\alpha} \\
X_{2}=X_{\beta}-A_{2} \cdot D_{\beta} \\
X_{3}=X_{\delta}-A_{3} \cdot D_{\delta} \\
X_{p}(t+1)=\left(X_{1}+X_{2}+X_{3}\right) / 3
\end{gathered}
$$

In the formula above, $X 1 \sim X 3$ represent the positions of three wolves respectively, and the positions of prey can be calculated according to formula (17). Then $\alpha, \beta$ and $\delta$ Wolf positions are updated according to the target position until the target is captured.

In GWO-SVM algorithm, penalty factor $c$ and kernel parameter $g$ are prey $X_{p}$ (i.e. optimization target), which is represented by the location of wolves closest to the target at the end of optimization. The following is a brief introduction of algorithm flow. First, sets the number $\mathrm{N}$ of the individual population and randomly generates the position ( the penalty factor $\mathrm{c}$ and the kernel parameter $\mathrm{g}$ ) of the grey wolf individual. The fitness function is defined as error rate, aiming at minimizing the error rate. The error rate is defined as:

$$
E(f, M)=\frac{1}{m} \sum_{i=1}^{m} \mathbb{I}\left(f\left(x_{i} \neq y_{i}\right)\right)
$$

where $M$ represents the sample set. In short, the error rate is the number of predicted results of the statistical classifier that are different from the real results, and then divided by the total number of sample set $M$. At last, a large number of segmented characters are sent to SVM for training, and optimized to the best result. the proposed GWO-SVM algorithm is shown in fig.2. 


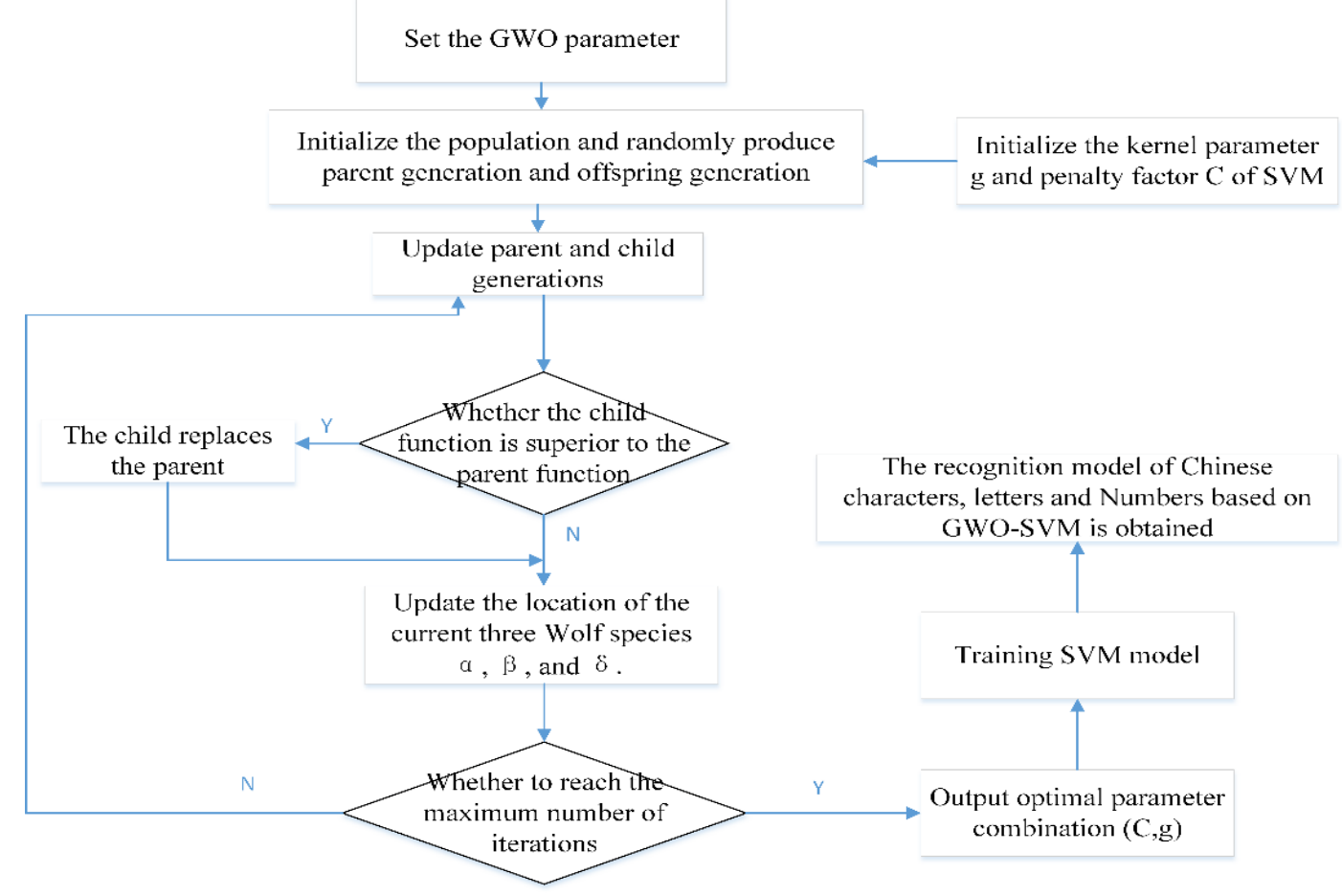

Fig. 2: The proposed GWO-SVM algorithm.

\section{Experimental Results and Analysis}

The experimental environment is based on Windows 7 (CPU / i5-4200 RAM / 4GB)/matlab 2017b and pycharm2019. The experimental data set consists of 5112 segmented Chinese license plate character images, which are divided into test set and training set according to 3:7 ratio. There are 1116 images of Chinese characters, 1693 images of English characters and 2303 images of digital characters.

The figure. 3 shows process of license plate recognition by the proposed method. The license plate is identified by the following operations: (1).Get original image as shown in Fig.3(a); (2).Using the proposed pre-processing method to get the gray level image as shown in Fig.3(b); (3).Mathematical morphology operation on gray level image after pre-processing is shown in Fig.3(c),(d); (4).Canny edge detection algorithm was used for edge detection, and then the image of edge detection was binarized as shown in Fig.3(e); (5).The opening and closing operation in mathematical morphology is used to connect the image into an overall picture as shown in Fig.3(f),(g); (6). The rectangular frame is located by prior knowledge such as color feature, license plate size, length width ratio of license plate and so on;(7).Using the perspective transformation to correct the license plate area;(8).Analysis of the grey image of license plate horizontal projection and vertical projection histogram peak and valley to determine the segmentation threshold to segment license plate characters as shown in Fig.3(i),(j). After the above character recognition, the license plate recognition model can accurately recognize the license plate and the recognition results is given including license plate color, recognition time, license plate number and other key information.

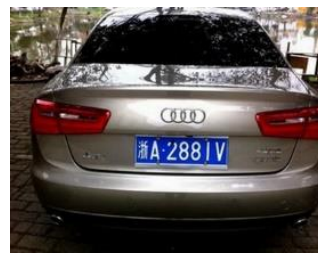

(a)

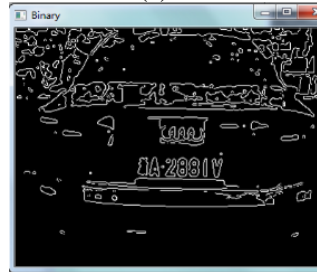

(e)

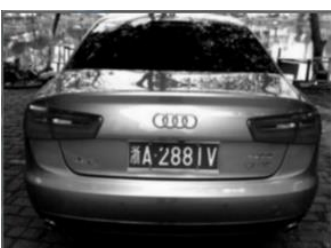

(b)

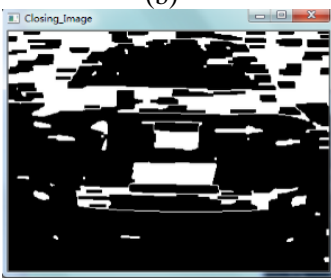

(f)

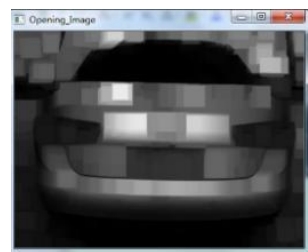

(c)

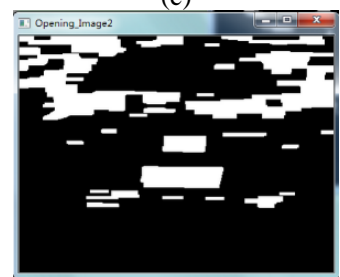

$(\mathrm{g})$

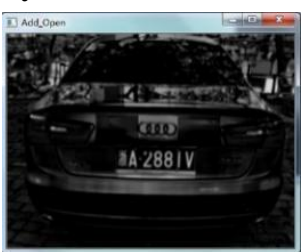

(d)

\section{EA288IV}

(h) 

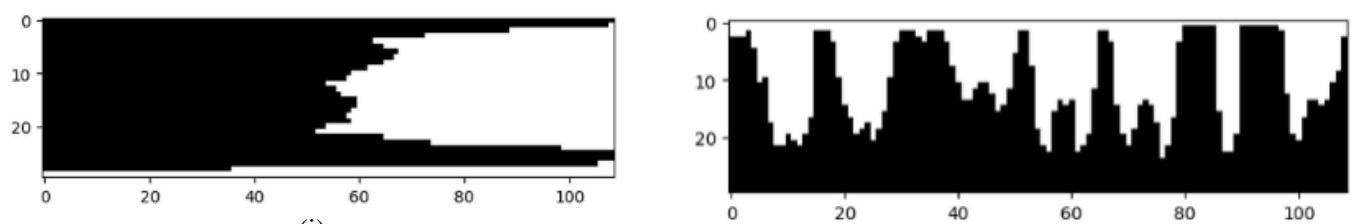

(i)

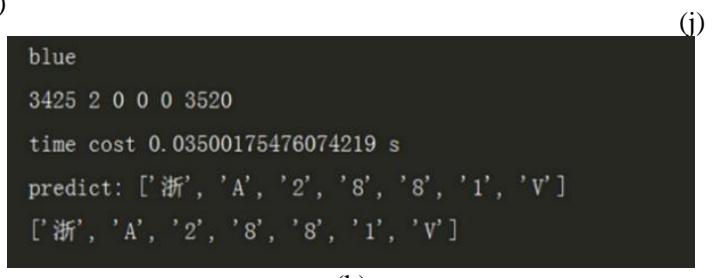

(k)

Fig. 3: The step and result of license plate recognition.

According to the characteristics of Chinese license plate characters, the license plate characters are composed of seven characters and can be divided into three categories: Chinese characters, letters and numbers. In this paper, the character recognizer is trained by the Histogram of Oriented Gradient (HOG) features of characters. Considering the differences between Chinese characters, letters and numbers, the character recognizer is trained separately. In order to verify the accuracy of the character recognition algorithm, the training set and the test set are in a ratio of 7:3. Character recognition accuracy is shown in the TABLE I.

Table 1: Results of Character Recognition

\begin{tabular}{ccc}
\hline \multicolumn{3}{c}{ Character Recognition } \\
\hline Category & accuracy & $\begin{array}{c}\text { Recognized } \\
\text { character } \\
\text { ratio }\end{array}$ \\
\hline $\begin{array}{c}\text { Chinese } \\
\text { Character }\end{array}$ & $95.79 \%$ & $1069 / 1116$ \\
\hline Letter & $99.57 \%$ & $1632 / 1693$ \\
\hline Digital & $99.65 \%$ & $2295 / 2303$ \\
\hline
\end{tabular}

From the result in TABLE I, we can see that the recognition accuracy of numbers is better than Chinese characters, due to the complexity of font structure. Chinese characters are more difficult to recognized than English letters and Arabic numerals, which is consistent with the experimental results.

In addition, SVM character recognition based on other optimization algorithms are trained, such as SVM based on genetic algorithm (GA-SVM), SVM based on particle swarm algorithm (PSO-SVM). The recognition accuracy of various algorithms is shown in TABLE II. And the character optimization time is shown in TABLE III.

Table 2: The Recognition Accuracy of Various Algorithms

\begin{tabular}{cccc}
\hline \multicolumn{4}{c}{ Character Recognition Accuracy (Data set:5112) } \\
\hline Algorithm & $\begin{array}{c}\text { Chinese } \\
\text { Character }\end{array}$ & Letter & Digital \\
\hline SVM & $86.00 \%$ & $92.00 \%$ & $95.00 \%$ \\
GA-SVM & $95.88 \%$ & $99.20 \%$ & $99.14 \%$ \\
PSO-SVM & $95.14 \%$ & $99.42 \%$ & $99.43 \%$ \\
GWO-SVM & $\mathbf{9 5 . 7 9 \%}$ & $\mathbf{9 9 . 5 7 \%}$ & $\mathbf{9 9 . 6 5 \%}$ \\
\hline
\end{tabular}

Table 3: The Character optimization time comparison table

\begin{tabular}{|c|c|c|}
\hline Algorithm & Operation time $(s)$ & Test Condition \\
\hline GA-SVM & 513.94 & Data set: 350 \\
\hline PSO-SVM & 298.13 & Maximum \\
\hline GWO-SVM & 104.05 & $\begin{array}{l}\text { iterations: } 20 \text {; } \\
\text { Population: } 10 \text {; }\end{array}$ \\
\hline
\end{tabular}


Seen from TABLE II and TABLE III, the accuracy of SVM recognition is greatly improved by various swarm intelligence optimization models, while the precision difference between optimization algorithms is not obvious. However, compared with other optimization algorithms mentioned above, the proposed GWOSVM algorithm has strong global optimization ability, fast iteration and fast searching speed.

\section{Conclusion}

In order to improve the efficiency of traditional SVM-based LPR, this paper proposes a lightweight LPR algorithm based GWO-SVM algorithm, which uses GWO to seek the optimal parameters of combined penalty factor $\mathrm{c}$ and kernel parameter $\mathrm{g}$ of SVM. In addition, based on the introduction of LPR general process, Gaussian filter and gray level stretching are introduced to the image pre-processing. Experimental results show that the recognition accuracy of the proposed character recognition model can reach than 95\%. Compared with other LPR models based on SVM, this algorithm has faster speed, and is suitable for lowcost equipment.

However, the proposed method has many constraints such as illumination, lens distortion. We plan to introduce the uneven illumination correction algorithm and the more effective tilt correction algorithm to solve the above problems. The further work will focus on the two aspects of plate accurate positioning and plate accurate correction.

\section{References}

[1] Shivakumara, P., Tang, D., Asadzadehkaljahi, M., Lu, T., Pal, U., \& Anisi, M. H. (2018). CNN-RNN based method for license plate recognition. CAAI Transactions on Intelligence Technology, 3(3), 169-175. doi:10.1049/trit.2018.1015

[2] Peiqi Wu, Zhangqin Huang and Da Li. Research on the character recognition for Chinese license plate based on CNN [C].3 $3^{\text {rd }}$ IEEE International Conference on Computer and Communications, 2017:1652-1656.

[3] GHAHNAVIEH A E, AMIRKHANI-SHAHRAKIA, RAIE A A.Enhancing the license plates character recognition methods by means of SVM [C]. 22nd Iranian Conference on Electrical Engineering (ICEE), 2014:220-225.

[4] Yu Y, Hong Z, Ting-Ting G, et al. Algorithm of License Plate Recognition Based on Joint HOG Feature[J]. Computer Engineering and Design, 2015, 36(2): 476-481.

[5] Khan, M. A., Sharif, M., Javed, M. Y., Akram, T., Yasmin, M., \& Saba, T. (2018). License number plate recognition system using entropy-based features selection approach with SVM. IET Image Processing, 12(2), 200209. doi:10.1049/iet-ipr.2017.0368

[6] Mirjalili S, Mirjalili S M, Lewis A. Grey Wolf Optimizer[J]. Advances in Engineering Software, 2014, 69(3): 4661.

[7] A. H. Ashtari, M. J. Nordin and M. Fathy, "An Iranian License Plate Recognition System Based on Color Features," in IEEE Transactions on Intelligent Transportation Systems, vol. 15, no. 4, pp. 1690-1705, Aug. 2014.

[8] Chanika Sukawattanavijit, Chen Jie, Zhang Hongsheng. GA-SVM algorithm for improving land-cover classification using SAR and optical remote sensing data [J]. IEEE Geoscience and Remote Sensing Letters, 2017, 14 (3):284-288.

[9] Seibel, H., Goldenstein, S., \& Rocha, A. (2017). Eyes on the Target: Super-Resolution and License-Plate Recognition in Low-Quality Surveillance Videos. IEEE Access, 5, 20020-20035. doi:10.1109/ACCESS.2017.2737418

[10] Yousif, B. B., Ata, M. M., Fawzy, N., \& Obaya, M. (2020). Toward an Optimized Neutrosophic k-Means With Genetic Algorithm for Automatic Vehicle License Plate Recognition (ONKM-AVLPR). IEEE Access, 8, 4928549312. doi:10.1109/ACCESS.2020.2979185 